\section{Compact, wideband impedance tuner using a three-line-microstrip structure}

\section{J. Heredia, M. Ribó and L. Pradell ${ }^{\bowtie}$}

A novel three-line-microstrip compact impedance tuner is presented. It is based on a multimodal structure wherein six variable capacitances, implemented with two parallel-connected varactors each, create multiple interactions among the three-line-microstrip modes in a reduced circuit area. Experimental results show better-than- $70 \%$ coverage of the Smith chart in an $85 \%$ frequency bandwidth from 1.4 to $3.2 \mathrm{GHz}$.

Introduction: Impedance tuners are extensively used to implement tunable matching networks in power amplifiers [1] or antennas [2], and for load-pull or noise-parameter characterisation [3]. To provide flexibility in reconfigurable systems, the tuner should be compact in size, with a wide Smith-chart coverage and large fractional frequency bandwidth (FBW). Recent MOS and BiCMOS mm-wave tuners [2, 3] exhibit a very small area, but limited (an estimated 35\%) Smith-chart coverage. In [4] a small-size, low-power-consumption tuner for RFID applications using varactors is proposed featuring a $50 \% \mathrm{FBW}$ (1.8-3 GHz), moderate (an estimated 64.3\%) Smith-chart coverage, with complete coverage of all reflection coefficients with voltage standing-wave ratio (VSWR) lower than $\mathrm{VSWR}_{\mathrm{CC}}=4.6$ (and any phase) at $2.2 \mathrm{GHz}$. The integrated-passive device tuner using ferroelectric varactors in [5] features small size, and a 40\% FBW (2-3 GHz) for an estimated $40 \%$ Smith-chart coverage and $\mathrm{VSWR}_{\mathrm{CC}}=1.8$. In [1] a substrate-integrated-cavity technology with piezoelectric actuated dishes is used to achieve a $37 \%$ FBW $(2.5-3.9 \mathrm{GHz})$ with a $50 \%$ Smith-chart coverage, and $\mathrm{VSWR}_{\mathrm{CC}}=2$ in the $2.53 .7 \mathrm{GHz}$ frequency range.

Multimodal circuits use multimodal waveguides to allow the propagation of more than one fundamental mode in the same circuit area The additional modes increase the equivalent electrical length of the circuit, allowing for designs that are more compact. Multimodal circuits have demonstrated compact size and reconfiguration capabilities in structures such as filters [6].

In this Letter, a novel varactor-loaded multimodal threeline-microstrip (TLM) structure is used to implement a wideband impedance tuner with a wide, uniform Smith-chart coverage in the whole operation band.

Tuner structure and implementation: The tuner structure is shown in Fig. 1. It consists of a TLM section connected to microstrip lines (to provide an easy access using microstrip-to-coaxial transitions), with two series gaps in its outer strips, and six variable capacitances. The TLM propagates three fundamental modes, the ee, oo, and oe modes [7] (roughly equivalent to the microstrip, coplanar-waveguide, and slotline modes). The microstrip modes basically generate ee modes at the microstrip-to-TLM transitions, which then excite (and afterwards interact with) the $o o$ and $o e$ modes at the gaps and capacitors, which in turn resonate in the TLM section. Thus, a rich resonant $o o-o e$ structure coexists with the exciting ee structure in the same physical circuit area, resulting in an increase of the equivalent electrical size of the circuit.

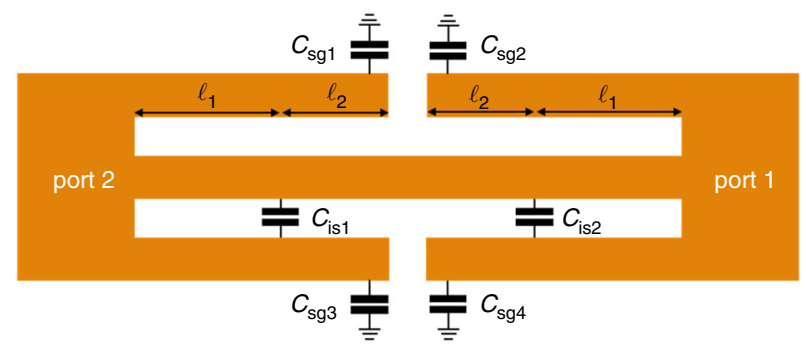

Fig. 1 TLM impedance tuner structure

To analyse the circuit mode interaction, the modal equivalent circuits proposed in [8], which transform the actual voltages and currents on generic $n$-conductor lines into their modal counterparts, can be used. This way the tuner states (reflection coefficients as a function of the variable capacitances) can easily be simulated at any given frequency. To achieve a compact circuit, the TLM total electrical length 2 . $\left(\ell_{1}+\ell_{2}\right)$ was limited to $\lambda / 8\left(45^{\circ}\right)$ at the design frequency $(2 \mathrm{GHz})$. Under this restriction, the relative positions of the two parallel inter-strip varactors $\left(\ell_{1}, \ell_{2}\right)$ were then optimised by a parametric study to achieve a maximal Smith-chart coverage, resulting in $\ell_{1}=1.48 \cdot \ell_{2}$. Fig. 2 compares the ideal (with lossless lines and a $0-3.9 \mathrm{pF}$ capacitance range) simulated results $\left(S_{11}\right)$ of the proposed tuner with those of a conventional tuner composed of a microstrip line of the same electrical length and the same number of shunt-connected variable capacitances uniformly distributed along the line. As can be seen, whereas the Smith-chart coverage achieved with the conventional tuner is moderate $(54 \%)$, the proposed tuner features a complete (100\%) coverage, which could only be achieved by a much larger $(1.5 \lambda)$ conventional structure.
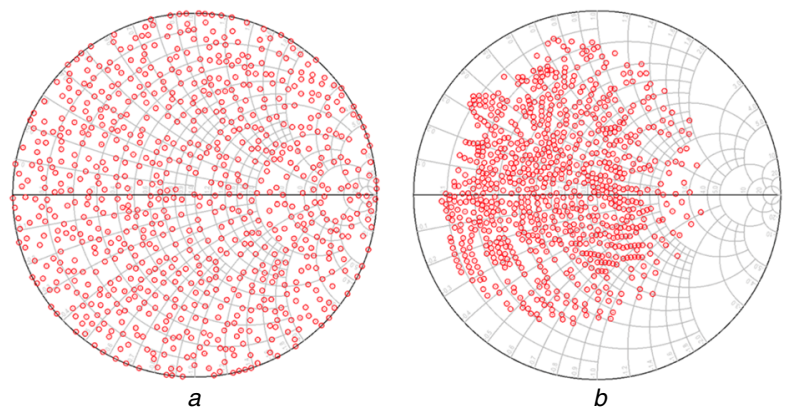

Fig. 2 Comparison of simulated tuner coverage $\left(S_{11}\right)$ at $2 \mathrm{GHz}$ using TLM and conventional microstrip structures

a Proposed tuner (Fig. 1)

$b$ Conventional microstrip tuner

Fig. 3 shows a picture of the fabricated tuner. It was implemented on a dielectric substrate with $\varepsilon_{\mathrm{r}}=3.55$, height $=0.81 \mathrm{~mm}$ and $\tan (\delta)=0.0022$. Variable capacitances were implemented with two parallel varactors (MACOM MA46580) to increase the capacitance range $(0.3-3.9 \mathrm{pF}$ for a bias voltage range of $18-0 \mathrm{~V})$. Parallel-plate capacitors and resistors were used to decouple the varactors in RF, islands on the lower strips to bias the $C_{\mathrm{is} 1}$ and $C_{\mathrm{is} 2}$ varactors, and via holes to connect the $C_{\mathrm{sg} 1}, C_{\mathrm{sg} 2}, C_{\mathrm{sg} 3}$, and $C_{\mathrm{sg} 4}$ varactors to ground. The TLM line has inner- and outer-strip widths of 0.5 and $0.6 \mathrm{~mm}$, a slot width of $0.3 \mathrm{~mm}$ and $\ell_{1}=3.28 \mathrm{~mm}$.

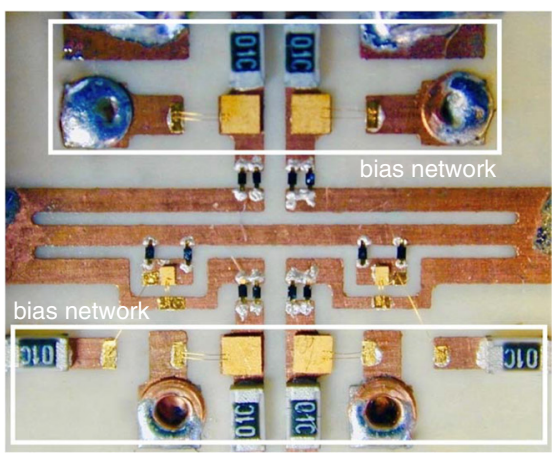

Fig. 3 Picture of fabricated TLM tuner

Experimental results: A total number of 1500 states were measured at $2 \mathrm{GHz}$, for a full capacitance-range variation of the varactors. Fig. 4 compares the simulated and measured results of the tuner input reflection coefficient $S_{11}$. The measured Smith-chart coverage is $73.2 \%$, and its $\operatorname{VSWR}_{\mathrm{CC}}$ is 6.2 . These results agree well with simulations $(76.3 \%$ Smith-chart coverage and VSWR $\leq 8.1)$. The error $\varepsilon$ between each simulated and measured state $i$ ( $i=1$ to 1500), defined as $\varepsilon=\left|S_{11 \text { (meas) }}^{i}-S_{11(\text { sim) }}^{i}\right|$, has a mean $m_{\varepsilon}=0.038$ and a standard deviation $s_{\varepsilon}=0.037$. Its histogram is shown in Fig. 5. The good agreement between simulation and measurement validates the multimodal analysis as a useful design tool for the proposed TLM tuner. To assess the tuner behaviour as a function of frequency, Figs. 6 and 7 compare the simulated and measured states at 1.4 and $3.2 \mathrm{GHz}$, respectively. The minimal measured Smith-chart coverage is $70 \%$ 
and its $\mathrm{VSWR}_{\mathrm{CC}}$ is 3.4 in the whole $1.4-3.2 \mathrm{GHz}$ frequency band (85\% FBW), and VSWR $\mathrm{VCC}_{\mathrm{CC}}>6.2$ for the $2-3.2 \mathrm{GHz}$ frequency band. The Smith-chart coverage, VSWR $\mathrm{CC}_{\mathrm{C}}$, and $\mathrm{FBW}$ of the proposed TLM tuner exceed those reported in [1-5]. It features a size of $0.13 \lambda \times 0.039 \lambda$, which is similar to that of the lumped-element tuner in [5] $(0.08 \lambda \times 0.044 \lambda)$, but smaller than that of the distributed-element tuners [1] $(0.56 \lambda \times 0.307 \lambda)$ and [4] $(0.25 \lambda \times 0.25 \lambda)$.
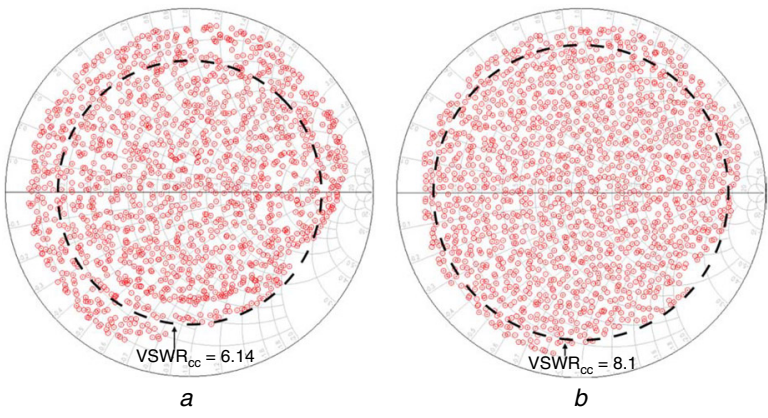

Fig. 4 Simulated and measured tuner coverage $\left(S_{11}\right)$ at $2 \mathrm{GHz}$

$a$ Measured tuner states

$b$ Simulated tuner states

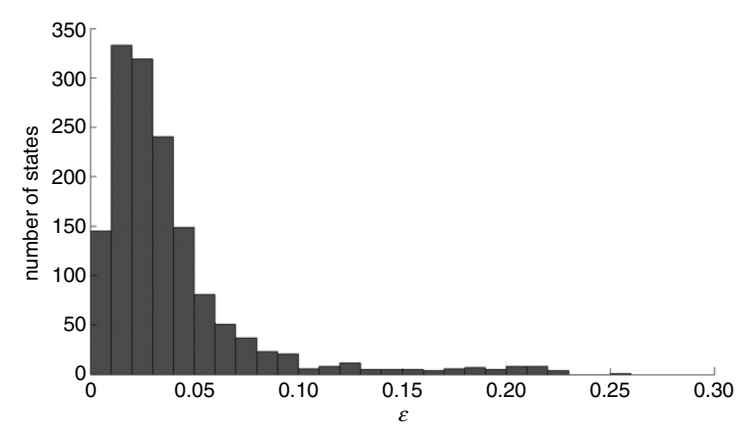

Fig. 5 Error histogram at $2 \mathrm{GHz}$
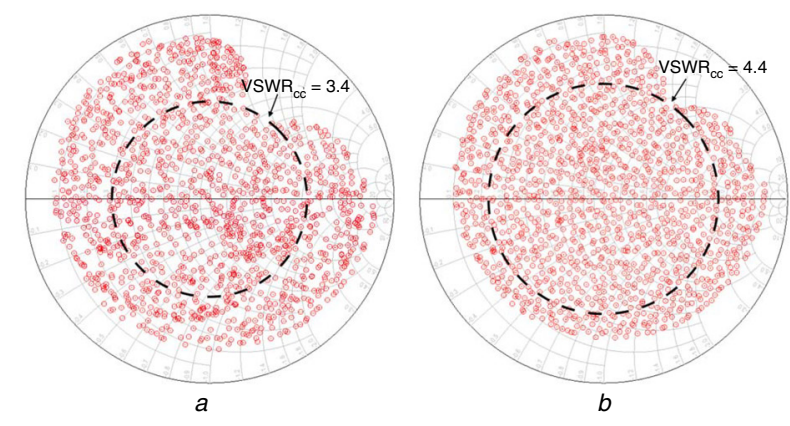

Fig. 6 Simulated and measured tuner coverage $\left(S_{11}\right)$ at $1.4 \mathrm{GHz}$

$a$ Measured tuner states

$b$ Simulated tuner states

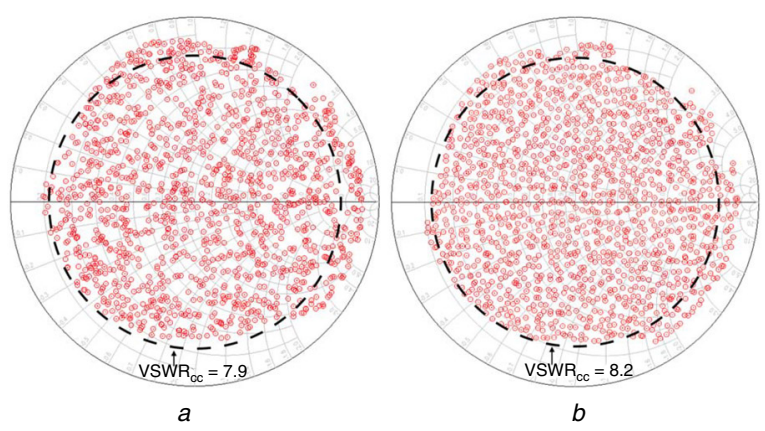

Fig. 7 Simulated and measured tuner coverage $\left(S_{11}\right)$ at $3.2 \mathrm{GHz}$

$a$ Measured tuner states

$b$ Simulated tuner states
Conclusion: A new kind of compact, wideband impedance tuner has been presented, simulated, fabricated, and measured. It is based on a multimodal TLM structure with six parallel-connected variable capacitances. It features a compact size of $0.13 \lambda \times 0.039 \lambda, 70 \%$ coverage of the Smith chart in an $85 \%$ FBW from 1.4 to $3.2 \mathrm{GHz}$, and a complete coverage of all reflection coefficients with VSWR $\leq 6.2$ and any phase in a $47 \% \mathrm{FBW}$ from 2 to $3.2 \mathrm{GHz}$.

Acknowledgments: This work was supported by the Spanish MEC under Projects TEC2013-48102-C2-1/2-P and TEC2016-78028C3-1-P, and grant no. BES-2011-051305, and the Unidad de Excelencia Maria de Maeztu MDM-2016-0600, which is financed by the AEI, Spain

(C) The Institution of Engineering and Technology 2018 Submitted: 19 February 2018 doi: $10.1049 / \mathrm{el} .2018 .0602$

One or more of the Figures in this Letter are available in colour online. J. Heredia and L. Pradell (Universitat Politècnica de Catalunya (UPC), TSC Dept, Campus Nord UPC, 08034 Barcelona, Spain)

凶-mail: pradell@tsc.upc.edu

M. Ribó (Electronics and Telecommunications Department, La SalleRamon Llull University (URL), Barcelona, Spain)

\section{References}

1 Semnani, A., Khater, M.A., Wu, Y.-C., et al.: 'An electronically tunable high-power impedance tuner with integrated closed-loop control', Microw. Wirel. Compon. Lett., 2017, 27, (8), pp. 754-756, doi: 0.1109/LMWC.2017.2723949

2 Vasilev, I., Lindstrand, J., Plicanic, V., et al.: 'Experimental investigation of adaptive impedance matching for a MIMO terminal with CMOS-SOI tuners', Trans. Microw. Theory Tech., 2016, 64, (5), pp. 1622-1633, doi: 10.1109/TMTT.2016.2546244

3 Bouvot, S., Bossuet, A., Quemerais, T., et al.: 'A D-band tuner for in-situ noise and power characterization in BiCMOS $55 \mathrm{~nm}$ '. IEEE Topical Meeting on Silicon Monolithic Integrated Circuits in RF Systems, Phoenix, AZ, USA, January 2017, pp. 103-106, doi: 10.1109/ SIRF.2017.7874384

4 Pochiraju, T., Fusco, V.F., Francey, J., et al.: 'Low-power variable impedance load/tuning unit', Electron. Lett., 2008, 44, (21), pp. 1258-1259, doi: 10.1049/el:20082283

5 Wong, K.W., and Mansour, R.R.: 'Impedance tuner using BST varactors in alumina-based IPD technology'. European Microwave Conf., London, UK, October 2016, pp. 1051-1054, doi: 10.1109/EuMC.2016.7824527

6 Contreras, A., Ribó, M., Pradell, L., et al.: 'K-band RF-MEMS uniplanar reconfigurable-bandwidth bandpass filter using multimodal immittance inverters', Electron. Lett., 2013, 49, (11), pp. 704-706, doi: 10.1049/ el.2013.0681

7 Tripathi, V.K.: 'Asymmetric coupled transmission lines in an inhomogeneous medium', Trans. Microw. Theory Tech., 1975, 23, (9), pp. 734-739, doi: 10.1109/TMTT.1975.1128665

8 Scanlan, J.O.: 'Theory of microwave coupled-line networks', Proc. IEEE, 1980, 68, (2), pp. 209-231, doi: 10.1109/PROC.1980.11618 